\title{
"Recovery of Kidney Function after 2 Years on Hemodialysis Due to Intra-renal Thrombotic Microangiopathy Induced by Interferon $\beta$-1a"
}

Short title: Late Recovery of Interferon-thrombotic Microangiopathy

\author{
Shaikha Al-Bader
}

MB BCh, MRCP (UK)

Department of Medicine, Al-Amiri Hospital, Kuwait

\section{Kamel El-Reshaid *}

MB BCh, Am B Med, Am B Nephrology, FRCP (Ed)

Department of Medicine, Faculty of Medicine, Kuwait University

\section{Corresponding Author -}

\section{Dr. Kamel El-Reshaid}

Professor, Department of Medicine, Faculty of Medicine, Kuwait University, P O Box 24923

\author{
13110 Safat, Kuwait $\quad$ Fax: (965) 25318454
}

E-mail: kamel @ hsc.edu.kw

\begin{abstract}
Beta interferons (IFN) are the first approved and the most-widely used disease modifying therapies for relapsing-remitting multiple sclerosis. Despite their good overall long-term safety data they can rarely cause renal thrombotic microangiopathy (TM). In this report, we describe a patient who developed a rapidly progressive renal failure with hypertension after receiving IFN- $\beta$-1a for 2 years. She had just mild anemia without laboratory evidence of hemolysis and her peripheral platelets counts were normal. Kidney biopsy was done despite her relatively smaller-sized kidneys at $8.8 \mathrm{~cm}$. It showed intra-renal acute on top of chronic TM. She did not have antiphospholipid antibodies, hypercoagulable state or genetic predisposition to TM. ADAMTS13 activity was normal. Despite discontinuation of IFN, plasma exchange and high dose corticosteroids for 2 weeks, she progressed fast to being dialysis-dependent. Her celiac and renal angiograms were normal. IFN $\beta-1 \mathrm{a}$ was replaced with Azathioprine and her severe hypertension was controlled. Interestingly, she remained dialysis-dependent for 2 years and subsequently, had recovered. One year later, her serum creatinine is at $129 \mathrm{umol} / \mathrm{L}$ and her kidneys size is $8 \mathrm{~cm}$. In-conclusion, IFN- $\beta$ treatment can induce renal-limited TM which may recover up to 2 years later.
\end{abstract}

Keywords: hypertension, interferon, multiple sclerosis, renal failure, thrombotic microangiopathy

\section{The case report:}

A 34-year-old Kuwaiti woman was referred for evaluation of renal failure with serum creatinine at 189 umol/L. The latter was diagnosed during routine work up for hypertension in the past 3 months. She was treated with Atenolol $100 \mathrm{mg}$ daily and had felt better. Past history was significant for remitting and relapsing MS and hence was treated with IFN $\beta$-1a (Rebif®, Merck Serono Europe Limited, London, UK) 2 years ago. Her initial examination was unremarkable except for blood pressure at 160/105 mm Hg and pallor yet without purpura. Systemic examination did not show abnormality. Laboratory investigations showed normal peripheral leukocyte and platelet counts. Hemoglobin was $90 \mathrm{~g} / \mathrm{L}$ with normal $\mathrm{MCV}$, transferrin saturation\%, vitamin B12, serum haptoglobin and $\mathrm{LDH}$. Serum urea was $16 \mathrm{mmol} / \mathrm{L}$ and creatinine at 189
umol/L. Serum glucose, electrolytes and liver functions were normal except for albumin at $30 \mathrm{~g} / \mathrm{L}$. Urine routine showed $2(+)$ proteinuria yet without hematuria or pyuria on microscopic examination. Twenty four hour urine collection revealed proteinuria at $2 \mathrm{~g} /$ day. Serum protein electrophoresis, complements $3 \& 4$, and IgA level were normal. ANA, anti-dsDNA, ANCA, anti-GBM antibodies, hepatitis $\mathrm{B}$ and $\mathrm{C}$ serology were negative. Abdominal and pelvic ultrasound did not show abnormality except for $8.8 \mathrm{~cm}$ kidneys with thin and echogenic cortex. Blood pressure was controlled after addition of Nifidipine retard $20 \mathrm{mg}$ twice daily and her anemia was corrected with erythropoietin. After control of hypertension, she was subjected to an ultrasound-guided kidney biopsy by an interventional radiologist. On microscopy, the biopsy showed 38 glomeruli, 4 of which were globally sclerosed. Most of the glomeruli were moderately shrunken yet without matrix 


\section{International Journal of Innovative Research in Medical Science (IJIRMS) Volume 03 Issue 09 Sept. 2018, ISSN: 2455-8737, Imp. Factor - 4.102 \\ Available online at $-\underline{w w w . i j i r m s . i n}$}

expansion, cellular proliferation, necrosis and crescent formation. Few showed segmental mesangiolysis. The glomerular capillary walls were unremarkable except for mild decrease in capillary lumens. On PAS stain, the capillary basement membranes showed extensive corrugation and some showed segmental splitting. One glomerulus showed thrombotic congestion/infarction with red cell engorgement and intra-capillary fibrin. The tubules and interstitium showed mild tubular atrophy and infiltration with lymphocytes and plasma cells near the scarred areas (fibrosis) which accounted for $60 \%$ of the cortical tissue. An arcute artery together with interlobular arteries and numerous small caliber arteries showed advanced luminal narrowing to occlusion, due to intimal fibrosis, smooth muscle proliferation and mucoid change together with lamellar intimal fibromuscular hyperplasia (Onion-skinning) with fibrin clot (Fig 1). In addition, a few interlobular arteries showed marked endothelial swelling associated with RBCs fragmentation. Immunoperoxidase stains showed focal and segmental coarse granular staining limited only to some peripheral capillary loops for IgM (3+), C1q (3+), C3 (2+), and fibrinogen (2+). Hence, the final report was consistent with acute on top of chronic TM that had involved the glomeruli together with arcuate, interlobular and small caliber arteries.

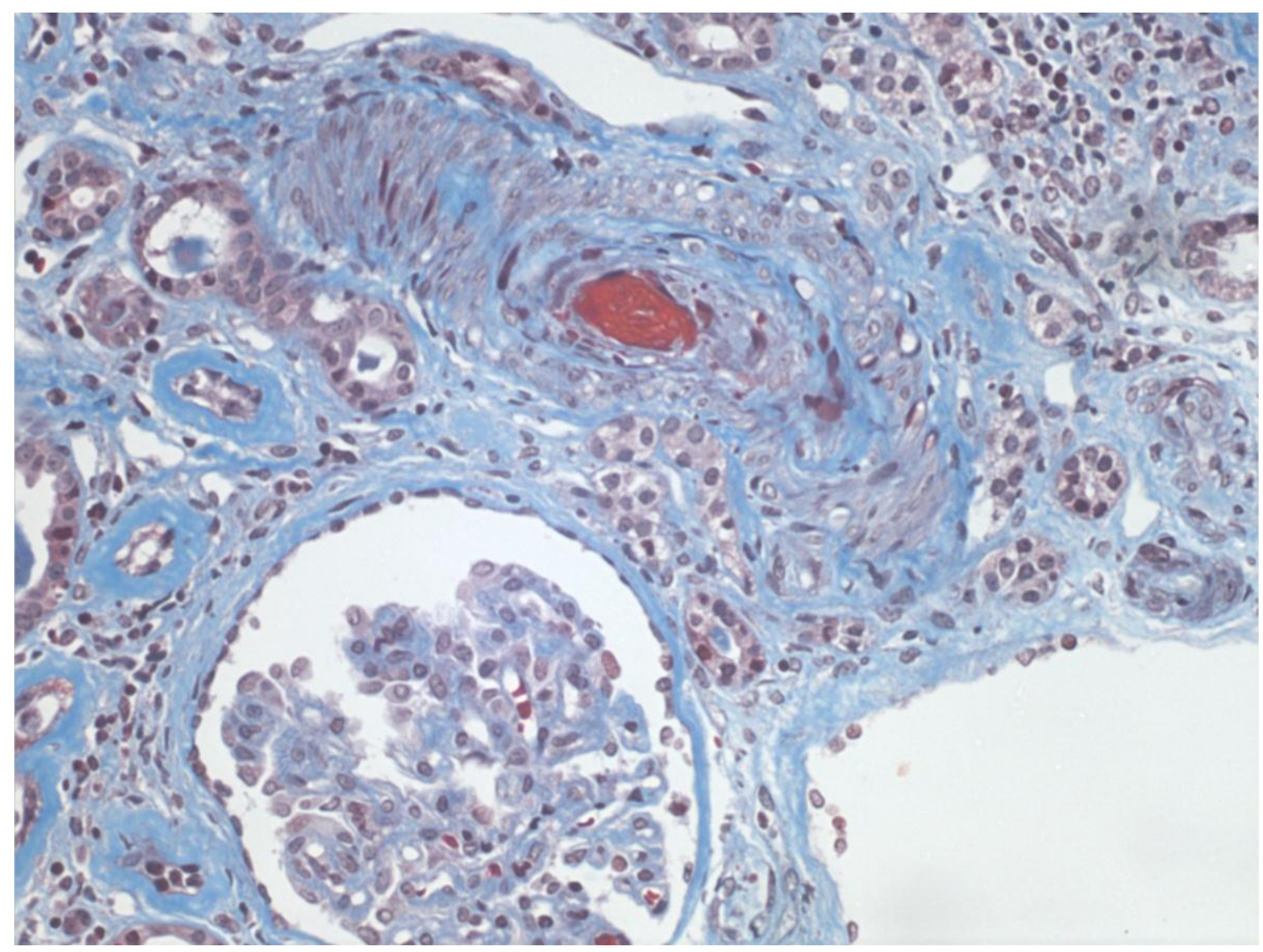

Figure 1: Photomicrograf of kidney biopsy showing a preserved glomerulus with mesangiolysis near an interlobular artery showing intimal fibromuscular hyperplasia (Onion-skinning) and a recent thrombus (Trichrome X100).

Despite discontinuation of IFN, plasma exchange and high dose corticosteroids, she progressed within 2 weeks to being dialysisdependent. Her accelerated hypertension was difficult to control and had required 5-drug combination. Since, she was dialysisdependent; Lisinopril was added at a dose of $20 \mathrm{mg}$ twice/daily. The latter improved her hypertension control and limited the drugs to Atenolol, Nifidipine retard and Lisinopril only. For her MS, she was treated with Azathioprine $75 \mathrm{mg}$ daily. Subsequently, there was no evidence of relapse of multiple sclerosis, accelerated hypertension and TM. She remained hemodialysis-dependent for 2 years. Subsequently, she gradually regained adequate function and dialysis was discontinued 1 year ago. By now her last serum creatinine is $129 \mathrm{umol} / \mathrm{L}$ and her last kidney ultrasound showed 8 $\mathrm{cm}$ kidneys with thin and echogenic cortex.

\section{Discussion}

TM has multiple etiologies viz. infections, malignancy, malignant hypertension, systemic lupus erythematosis, vasculitis, antiphospholipid syndrome, drug-induced, genetic predisposition with abnormalities in the complement and activity of von Willbrand protease factor (deficiency in ADAMTS) ${ }^{[10]}$ Our patient had acute on top of chronic renal disease and his kidney biopsy was consistent with TM. Her hypertension was only for 3 months with normal celiac and renal arteriography and a kidney biopsy showing TM rather than malignant changes. She did not have clinical, hematological, biochemical, serological and radiological evidence of previous co-morbid condition or genetic predisposition. The only drug taken was IFN $\beta-1$ a. Several hypotheses have been proposed to explain the mechanisms of TM with IFN therapy, but none is applicable in this case. The most plausible pathogenesis is inhibition of vascular endothelial cell growth factor (VEGF) in renal podocytes. VEGF stimulates signal transduction pathways and transcriptional programs through activation of its receptor (VEGFR2). ${ }^{[11]}$ These events are essential for de novo formation of blood vessels (i.e., angiogenesis, a process that involves proliferation and migration of endothelial cells). The latter process is inhibited by cytokines of the type 1 IFN family (including IFN- $\alpha / \beta) .{ }^{[12]}$ IFN- $\alpha / \beta$ exerts their effects via binding to the type 1 IFN receptor and activation of Janus kinases 
and signal transducers, and activators of transcription. ${ }^{[13]}$ The role of VEGF in renal physiology is not completely known, but it has been involved in the pathophysiology of several renal diseases, and $\mathrm{TM}$ is a lesion commonly reported with anti-VEGF drugs. ${ }^{[14]}$ Hence, IFN therapy can mediate TM through this antiangiogenic activity. The association of INF with TM has been defined in a study showing a clinical phenotype of cases referred to a national center had uniformly consistent direct dose-dependent druginduced model of INF toxicity. It included specific microvascular pathological changes seen in patient biopsies, and were dependent on transcriptional activation of the IFN response through the type I IFN receptor (IFNAR). ${ }^{[15]}$

What is unique in our patient is the late recovery of severe renal failure. This entails; (a) Lack of the rapid improvement (within days-weeks) in kidney function after discontinuation of IFN $\beta$-1a seen in previous reports. (b) Late recovery of reasonable kidney function 2 years after being dialysis-dependent. The answer may be related to different phenotype-predisposition to IFN $\beta$ - 1 a similar to the phenomenon of IFN $\beta$-1a induced TM in few patients while millions had received the drug and for years without similar side effect. ${ }^{[15]}$ Moreover, though our patient had severe renal failure; her kidney biopsy showed modest chronicity index and her blood pressure was controlled well after addition of an angiotensin converting enzyme inhibitor similar to the phenomenon seen in scleroderma crisis. ${ }^{[16]}$

In conclusion, IFN therapy should be stopped at the earliest signs of renal derangement in susceptible patients viz. hypertension and proteinuria to avoid long-term complications. Moreover, nephrologists usually lose hope with persistent end-stage kidney disease for $>3$ months and ACEI should be used in this setting.

\section{References}

[1] Parkin J, Cohen B. An overview of the immune system. Lancet 2001; 357: 1777-1789.

[2] Jadoul M, Piessevaux H, Ferrant A, Cosyns JP, van Ypersele de Strihou C. Renal thrombotic microangiopathy in patients with chronic myelogenous leukaemia treated with interferon-alpha 2b. Nephrol Dial Transplant 1995; 10: 111-113.

[3] Fabrizi F, Aghemo A, Fogazzi GB, Moroni G, Passerini P, D'Ambrosio R, Messa P. Acute tubular necrosis following interferon-based therapy for hepatitis $\mathrm{C}$ : case study with literature review. Kidney Blood Press Res 2013; 38: 52-60.

[4] Averbuch SD, Austin HA, Sherwin SA. Acute interstitial nephritis with the nephrotic syndrome following recombinant leukocyte A interferon therapy for mycosis fungoides. New Engl J Med1984; 310: 32-35

[5] Selby P, Kohn J, Raymond J, Judson I, McElwain T: Nephrotic syndrome during treatment with interferon. BMJ 1985; 290: 1180.

[6] Shah M, Jenis EH, Mookerjee BK, Schriber JR, Baer MR, Herzig GP, Wetzler M. Interferon- $\alpha$-associated focal segmental glomerulosclerosis with massive proteinuria in patients with chronic myeloid leukemia following high dose chemotherapy. Cancer1998; 83: 1938-1946

[7] Herrman J, Gabriel F. Membranoproliferative glomerulonephritis in a patient with hairy-cell leukemia treated with alpha-II interferon. N Engl J Med1987; 316: 112-113.

[8] Creutzig A, Freund M, Caspary L, Alexander K. Microangiopathy in patients with chronic myelogenous leukemia treated with interferon. Microvasc Res1996; 52: 288-292

[9] Hunt D, David Hunt, Kavanagh D, Iain Drummond I. et al. Thrombotic Microangiopathy Associated with Interferon Beta. N Engl J Med 2014; 370: 1270-1271.

[10] Moake JL. Thrombotic microangiopathies. N Engl J Med 2002; 347: 589-600.

[11] Ferrara N. Vascular endothelial growth factor: basic science and clinical progress. Endocr Rev 2004; 25: $581-$ 611.

[12] Sidky YA, Borden EC. Inhibition of angiogenesis by interferons: effects on tumor- and lymphocyte-induced vascular responses. Cancer Res 1987; 47: 5155-5161.

[13] Aaronson DS, Horvath CM. A road map for those who don't know JAK-STAT. Science 2002; 296: 1653-1655.

[14] 21. Schrijvers BF, Flyvbjerg A, De Vriese AS. The role of vascular endothelial growth factor (VEGF) in renal pathophysiology. Kidney Int 2004; 65: 2003-2017.

[15] Kavanagh D, McGlasson S, Jury A, et al. Type I interferon causes thrombotic microangiopathy by a dosedependent toxic effect on the microvasculature. Blood 2016; 128: 2824-2833.

[16] Guillevin L, Berezena A, Seror R, et al. Scleroderma renal crisis: a retrospective multicenter study on 91 patients and 427 controls. Rheumtology 2012; 51: 460467. 